PNL-SA-21741

\title{
DIRECT IONIZATION OF DNA IN SOLUTIONS
}

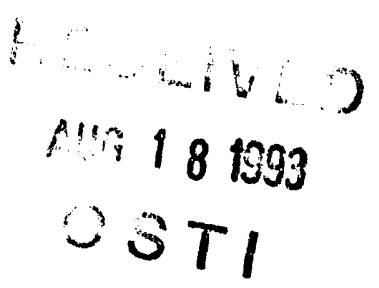

J. H. Miller

W. E. Wilson

R. H. Ritchie

\section{DISCLAIMER}

JuTy 1993

This report was prepared as an account of work sponsored by an agency of the United States Government. Neither the United States Government nor any agency thereof, nor any of their employees, makes any warranty, express or implied, or assumes any legal liability or responsibility for the accuracy, completeness, or usefulness of any information, apparatus, product, or process disclosed, or represents that its use would not infringe privately owned rights. Reference herein to any specific commercial product, process, or service by trade name, trademark, manufacturer, or otherwise does not necessarily constitute or imply its endorsement, recommendation, or favoring by the United States Government or any agency thereof. The views and opinions of authors expressed herein do not necessarily state or reflect those of the United States Government or any agency thereof.

Presented at the DOE Workshop on Computational Approaches in Molecular Radiation Biology \& Monte Carlo Methods Aprit 27-29, 1993

Irvine, California

Prepared for the U.S. Department of Energy Contract DE-AC06-76RLO 1830

Pacific Northwest Laboratory Richland, Washington 99352 


\title{
DIRECT IONIZATION OF DNA IN SOLUTION
}

\author{
J.H. Miller ${ }^{1}$, W.E. Wilson ${ }^{1}$, R.H. Ritchie ${ }^{2}$ \\ ${ }^{1}$ Pacific Northwest Laboratory, Richland, WA 99352, USA \\ ${ }^{2}$ Oak Ricge National Laboratory, Oak Ridge, TN 37831, USA
}

\begin{abstract}
Most of the energy absorbed in the cell nucleus from a radiation field goes into the aqueous medium that surrounds macromolecules, like DNA, which are critical to the normal function of cells. This part of the energy deposition produces numerous reactive species that can diffuse to DNA sequences and induce chemical changes. The average diffusion distance of the free radicals that mediate this indireci mode of DNA damage is only a few nanometers because the cellular medium contains a high concentration of molecules that rapidly scavenge the radiation-induced species. Under these conditions, direct interaction of the radiation field with DNA can not be neglected as a potential mode of damage induction. Two aspects of the direct effect will be discussed in this paper: (1) screening of the interaction between DNA and charged particles by the dielectric response of the aqueous medium and (2) the impact-parameter dependence of these interactions.
\end{abstract}

\section{INTRODUCTION}

Technical advances in molecular biology have made it possible to construct chemically well-defined DNA targets (oligonucleotides, plasmids, mini-chromosomes, etc.) for radiation studies. Use of these targets in traditional radiolysis experiments, (dilute aqueous solutions exposed to low LET radiation) has the potential to reveal many interesting aspects of the interaction of macromolecules with homogeneously distributed free radicals that escape from the tracks of charged particles slowing down 
developed by Bohr ${ }^{(4)}$, one expects that components that are close to the particle trajectory and that have low-energy excitation modes with high oscillator strength will have the greatest probability of receiving energy from a fast charged projectile.

In this paper we will describe calculations of cross sections differential in the energy of secondary electrons for direct ionization of DNA in solution and the semiclassical representation of these cross sections as an integral over impact parameters. Calculations of this type, tested whenever possible by experimental data, should provide the imput data needed for track-structure simulations of DNA damage by direct interaction with charged particles in a radiation field.

\section{METHODS}

The first Born approximation for the differential cross section to eject electrons from absorption band $i$ of a homogeneous condensed medium by energy transfer $h \omega$ from a fast particle with nuclear charge $Z$ and kinetic energy $E$ is ${ }^{(5)}$

$$
\pi a_{0} n_{1} E \frac{d \sigma_{1}}{d(\hbar \omega)}=\int_{k_{\min }}^{k_{\max }} \operatorname{Im}\left[\epsilon_{1}(k, \omega)\right] \frac{z^{2}}{|\epsilon|^{2}} \frac{d k}{k}
$$

where $a_{0}$ is the Bohr radius $(0.0529 \mathrm{~nm})$ and $n_{i}$ is the density of electrons involved in the transition that gives rise to the $\mathrm{i}^{\text {th }}$ absorption band. The integral is over the range of momentum transfer allowed by the conservation of energy and momentum in the collision. $\operatorname{Im}\left[\epsilon_{i}(k, \omega)\right]$ is the contribution from the $i^{\text {th }}$ band to the imaginary part of the complex dielectric response function $\epsilon$ and is analogous to the generalized oscillator strength of an electronic subshell of an atom or molecule in the gas phase.

The extension of this theory to a heterogenous medium is trivial when the macromolecular solute is present in such low concentration that its effect on the bulk dielectric response function is negligible. In this case, $i$ denotes an impurity band in the medium, $\operatorname{Im}\left[\epsilon_{i}\right]$ is proportional to the generalized oscillator strength of the band, and $|\epsilon|^{2}$ is the square modulus of the complex dielectric response function of the medium in the absence of the impurity. At this level of approximation, the impurity is treated as a gas-phase molecule with a solvation structure.

The effects of collective modes of excitation in the medium, if they are present, are contained in $|\epsilon|^{2}$. The primary role of the bulk medium in the collision process can be interpreted as an effect on the electromagnetic field of the projectile. Localized electronic polarization of the medium by the projectile may give rise to the condition $|\epsilon|^{2}>1$; then screening of the impurity site from the projectile occurs in a manner that is somewhat analogous to the effect of bound electrons on a projectile in the gas phase. Collective modes of excitation of the bulk medium by the projectile may be characterizes roughly by the condition $|\epsilon|^{2}<1$. This anti-screening effect can be interpreted as an electronic polarization wave excited in the bulk medium that may excite an electron of the impurity site ${ }^{(6)}$.

The general approach to obtaining an impact-parameter representation of collision cross sections involves the application of Fourier-transform analysis to 
spectrum, then a sum of terms like Eq.(4) is fitted to the experimental photoabsorption data. After the parameters of the OPWD model have been determined from the optical limit, the full dependence of $\operatorname{Im}[\epsilon]$ on momentum and energy transfer can be calculated from a somewhat more complicated analytic function ${ }^{(11)}$. The real part of the dielectric response function is evaluated numerically using the Kramers-Kronig ${ }^{(12)}$ relationship. It should be noted that the OPWD model is constrained to satisfy the Bethe sum rule.

\section{RESULTS}

Fig.(1) shows the result of fitting the liquid-water photoabsorption data ${ }^{(9)}$ by a sum of four terms like Eq.(4). Theoretical methods based on sum rules were

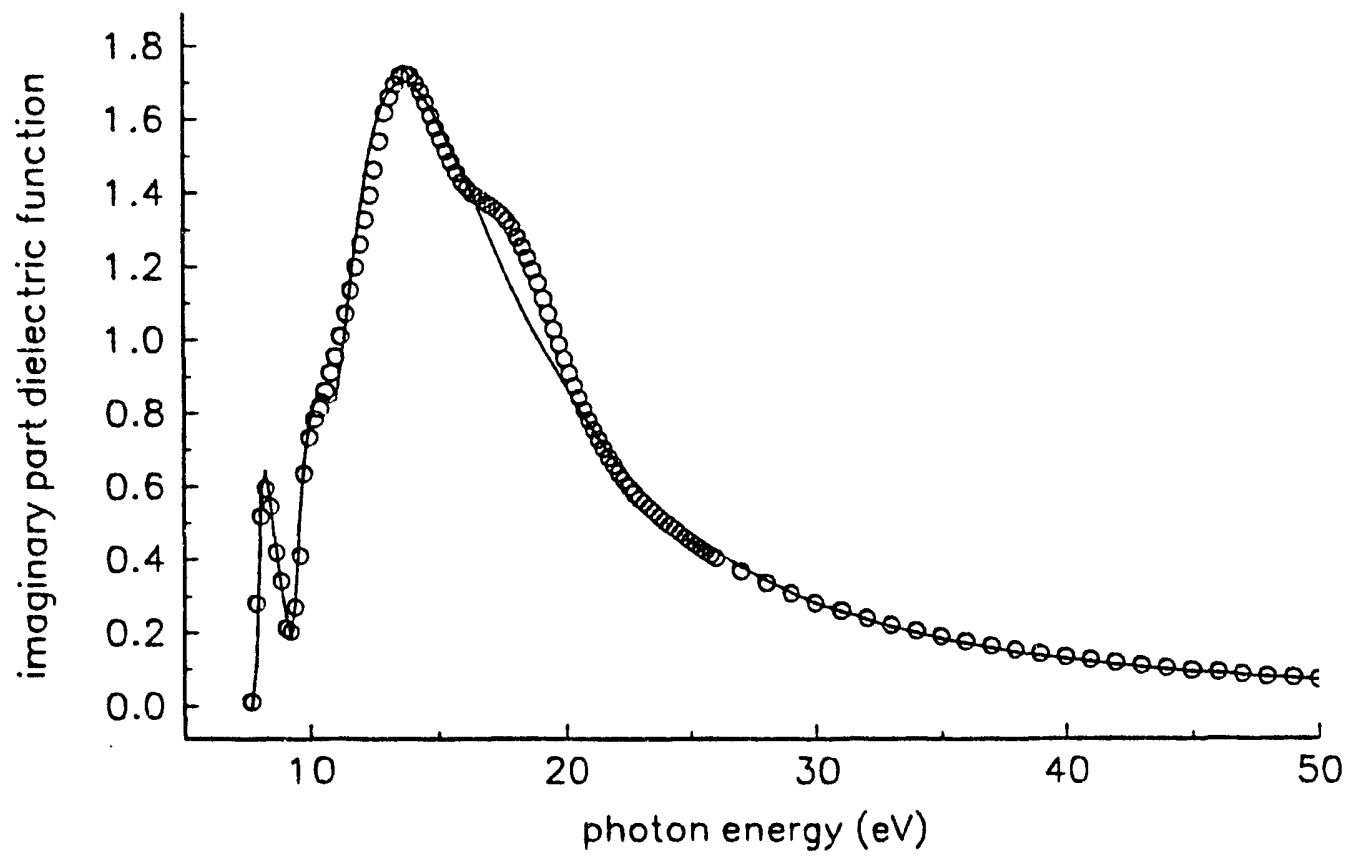

Figure 1. Fit of the OPWD model (solid curve) to liquid-water photoabsorption data (open circles) from ref.(9).

applied by Painter and coworkers ${ }^{(13)}$ to extend the experimental data from $25.5 \mathrm{eV}$ to $100 \mathrm{eV}$. The optimum parameter values are given in Table I. The parameters of the model are strongly coupled and the optimum values given in Table $I$ are not unique. Hence, little, if any, physical interpretation should be given to their value. A similar approach to obtaining the dielectric response function of liquid water has been carried out by others ${ }^{(14)}$. An alternative scheme for representing $\epsilon(k, \omega)$ for water based on the same optical data ${ }^{(9)}$ was incorporated early on into the Oak Ridge Monte Carlo code OREC ${ }^{(15)}$. 
Table 1. Parameters for the OPWD model of liquid water.

\begin{tabular}{cccc}
\hline Peak \# & Threshold (eV) & Alpha (au) & Eta (au) \\
\hline 1 & 7.76 & 0.285 & 0.00047 \\
2 & 9.25 & 0.531 & 0.0030 \\
3 & 10.88 & 0.880 & 0.024 \\
4 & 16.33 & 1.764 & 0.013
\end{tabular}

Photoabsorption data of $\mathrm{DNA}^{(10)}$ were multiplied by the ionization efficiency ${ }^{(16)}$ to obtain the experimental photoionization data for DNA that are shown by the open circles in Fig.(2). The solid curve in this figure is a fit to these data by Eq.(4) with

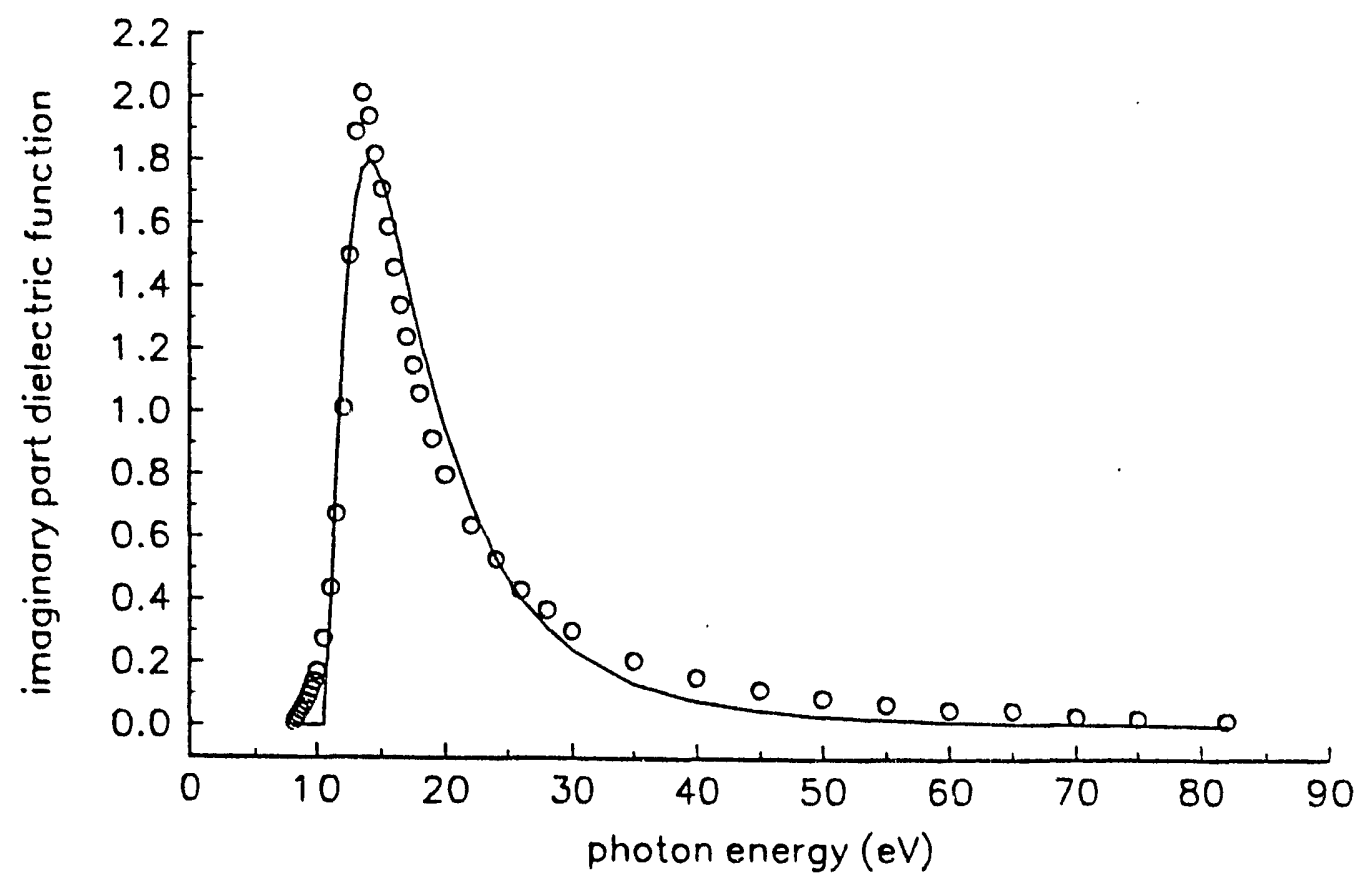

Figure 2. Fit of OPWD model (solid curve) to DNA photoionization data (open circles) from refs.(10) and (16).

an ionization threshold of $10.6 \mathrm{eV}, \alpha=0.875 \mathrm{au}$, and $\eta=0.0289$ au. Using these parameters to evaluate $\operatorname{Im}\left[\epsilon_{i}(k, \omega)\right]$ in Eq.(1) and the parameters in Table 1 to evaluate $|\epsilon|^{2}$, we obtain the differential ionization cross sections shown by the filled circles in Fig.(3) for a primary electron with a velocity of 3 au $(122.5 \mathrm{eV})$. To illustrate the effects of the solvent, the open circles show the result obtained for the same primary- and secondary-electron energies but with $|\epsilon|^{2}=1$. Clearly, the screening effect of the solvent dramatically reduces the cross section for ejection of low-energy secondary electrons and shifts the peak in the spectrum to a higher energy $(17)$. The small anti-screening effect for energy transfers between about 25 and 
$50 \mathrm{eV}$ is consistent with the general finding that collective modes of excitation in liquid water have a broad energy spectrum and short lifetime ${ }^{(18)}$.

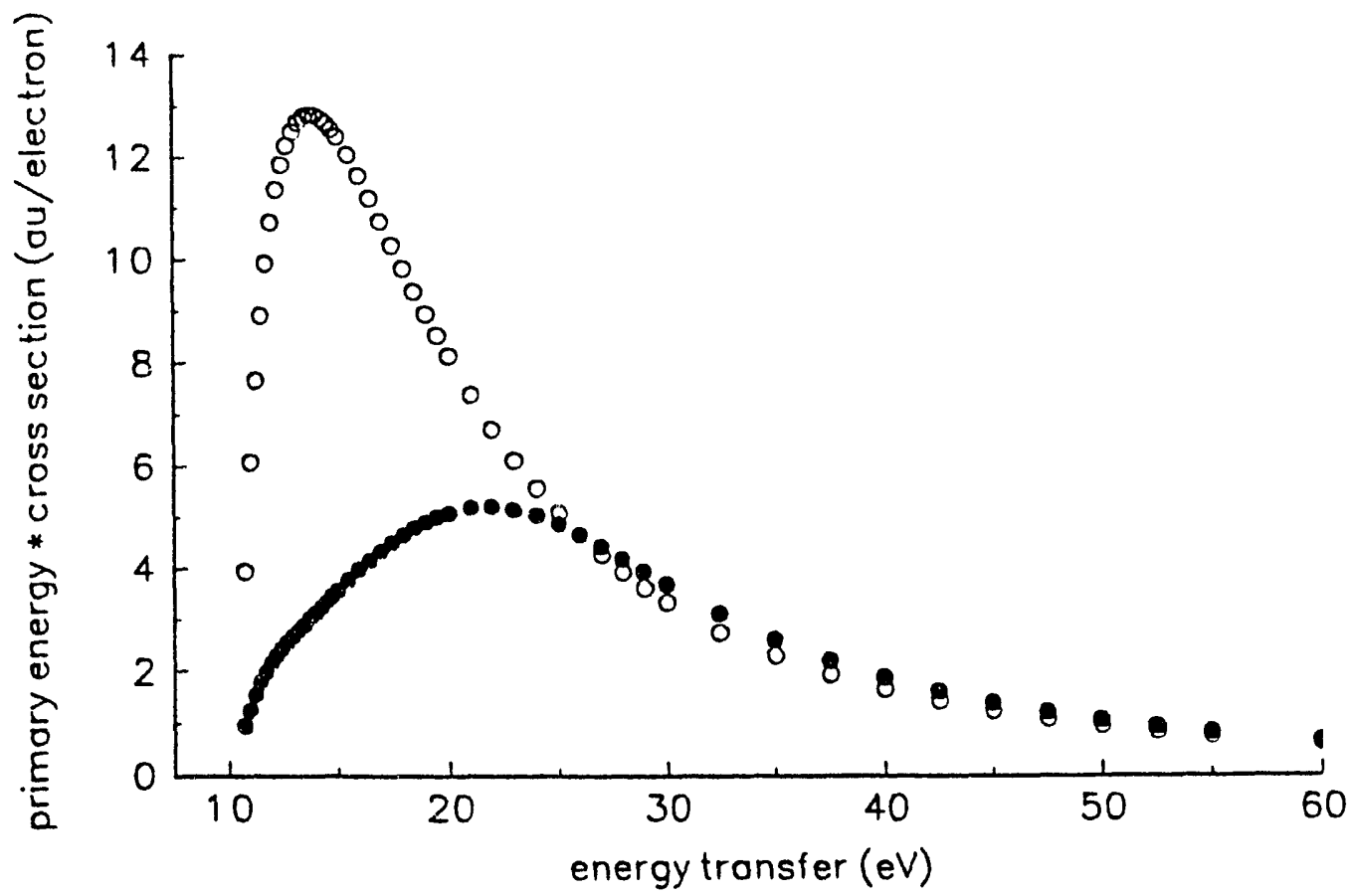

Figure 3. Differential cross sections for ionization of DNA with (solid) and without (open) screening by a water medium. The primary-electron energy is $122.5 \mathrm{eV}$.

Impact-parameter probability densities calculated from Eq.(2) with the normalization $\mathrm{dP} / \mathrm{db}=\left(\mathrm{d}^{2} \sigma_{\mathrm{i}} / \mathrm{d} \omega \mathrm{db}\right) /\left(\mathrm{d} \sigma_{\mathrm{i}} / \mathrm{d} \omega\right)$ are shown in Fig. (4) for ionization of DNA by $122.5 \mathrm{eV}$ primary electrons. The following levels of energy transfer are illustrated: (1) $11 \mathrm{eV}$ which is slightly above the threshold for ionization, (2) $22 \mathrm{eV}$ which is near the maximum in the differential energy-transfer cross section (see Fig.(3)), and (3) $54 \mathrm{eV}$ which is close to the energy transfer needed to produce the maximum secondary-electron energy in the usual convention of track simulation that the more energetic electron after an electron-impact ionization is the primary. The probability distributions calculated in the second and third cases conform to the expectation that as Bohr's maximum effective impact parameter, $v / \omega$, decreases, larger impact parameters become less efficient in collisional energy transfer. However, collisions with energy transfers near the ionization threshold appear to be an exception to this rule. Even though the maximum effective impact parameter is twice as large, the distribution of impact parameters in collisions where a $122.5 \mathrm{eV}$ primary electron transfers $11 \mathrm{eV}$ to the target is slightly more concentrated at small values than for collisions where $22 \mathrm{eV}$ of energy is transferred. This effect is probably a consequence of the energy- and momentum-transfer dependence of the dielectric functions used in Eq.(2). 


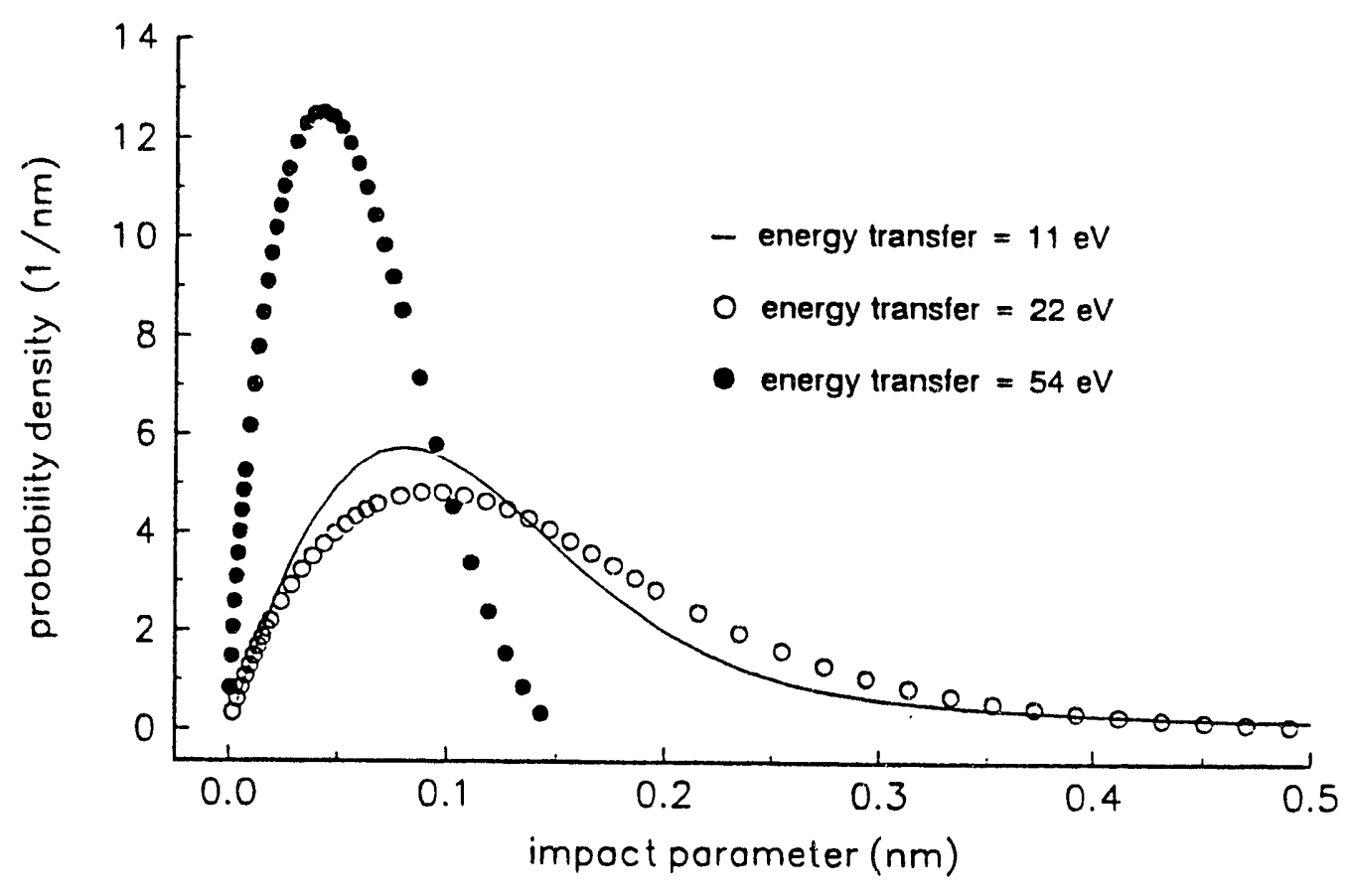

Figure 4. Impact-parameter distributions for three levels of energy transfer in ionizing collisions of DNA in water with $122.5 \mathrm{eV}$ primary electrons.

\section{DISCUSSION}

Our calculations show that Bohr's maximum effective impact parameter, $b_{c}=v / \omega$, determines the scale of the impact-parameter distribution in most cases. The probability to select an impact parameter much larger than $b_{c}$ in a Monte Carlo simulation of inelastic collisions will be very small. Hence, it is useful to compare $b_{c}$ with the characteristic size of the target of interest, the DNA double helix. This comparison is shown in Fig.(5) as a function of primary-electron energy for two levels of energy transfer. The filled circles show the ratio of the maximum effective impact parameter to the DNA radius $(1.23 \mathrm{~nm})$ for transfer of energy that is just sufficient to ionize the target (10.6 eV in our model of the DNA photoionization spectrum). The open circles show the ratio $b_{c} / 1.23 \mathrm{~nm}$ for the average energy transfer in an ionizing collision by a primary electron at the specified energy. Our results discussed above for $122.5 \mathrm{eV}$ primary electrons suggest that the closed circles in Fig.(5) over estimate of the scale of the impact-parameter distribution in collisions that produce very low-energy secondary electrons. In general, Fig.(5) suggests that the scale of the impact parameter distribution is comparable to or smaller than the DNA radius for many of the electron energies that are encountered in radiation fields of radiological interest. 


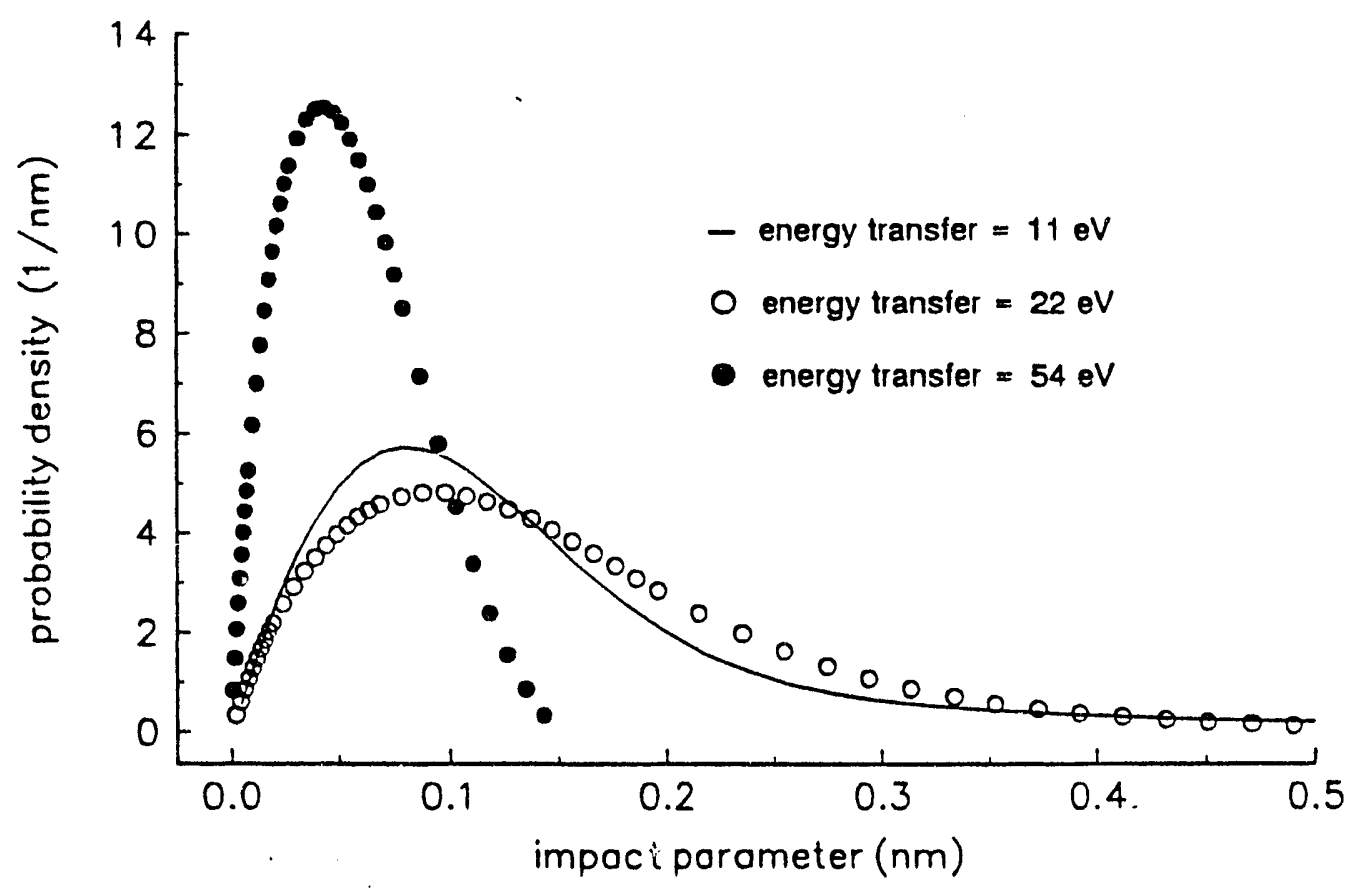

Figure 5. Ratio of the maximum effective impact paramuter to the DNA radius for ionizing collisions with primary electrons of different energy.

A cylinder with a $1: 23 \mathrm{~nm}$ radius centered on the helical axis contains not only the atoms of DNA in the standard $B$ conformation but also most of the water in the first two hydration shells, about 80 water molecules per nucleotide pair ${ }^{(19)}$. The consequences of ionizing an atom in this volume are probably unaffected by the presence of radical scavengers in the medium; hence, the results presented in this paper that ignore the finite size and internal structure of the target may be useful in estimating the non-scavengeable component of DNA damage for comparison with experiments ${ }^{(20)}$. However, the atoms within this volume are not equivalent with respect to the probability that ionization leads to a specific product. For example, ionization of a sugar moiety is more likely to result in strand scission than ionization of a base. Furthermore, the recent data obtained by Wheeler and coworkers ${ }^{(3)}$ with DNA at various levels of hydration suggest that ionization of water in the first hydration shell frequently leads to charge transfer to DNA bases while ionization of water in the second hydration shell appears to induce free radicals similar to those that result from ionization of bulk water.

Fig.(6) illustrates the next level of theoretical development that we feel is needed to address questions concerning observable products that result from direct interaction between DNA and charged particles in a radiation field. This extension of the present model would include the internal structure of DNA by identifying the functional groups that make up the polymer (phosphate, sugar, and the DNA bases adenine, guanine, cytosine and thymine). We propose to develop an impact 


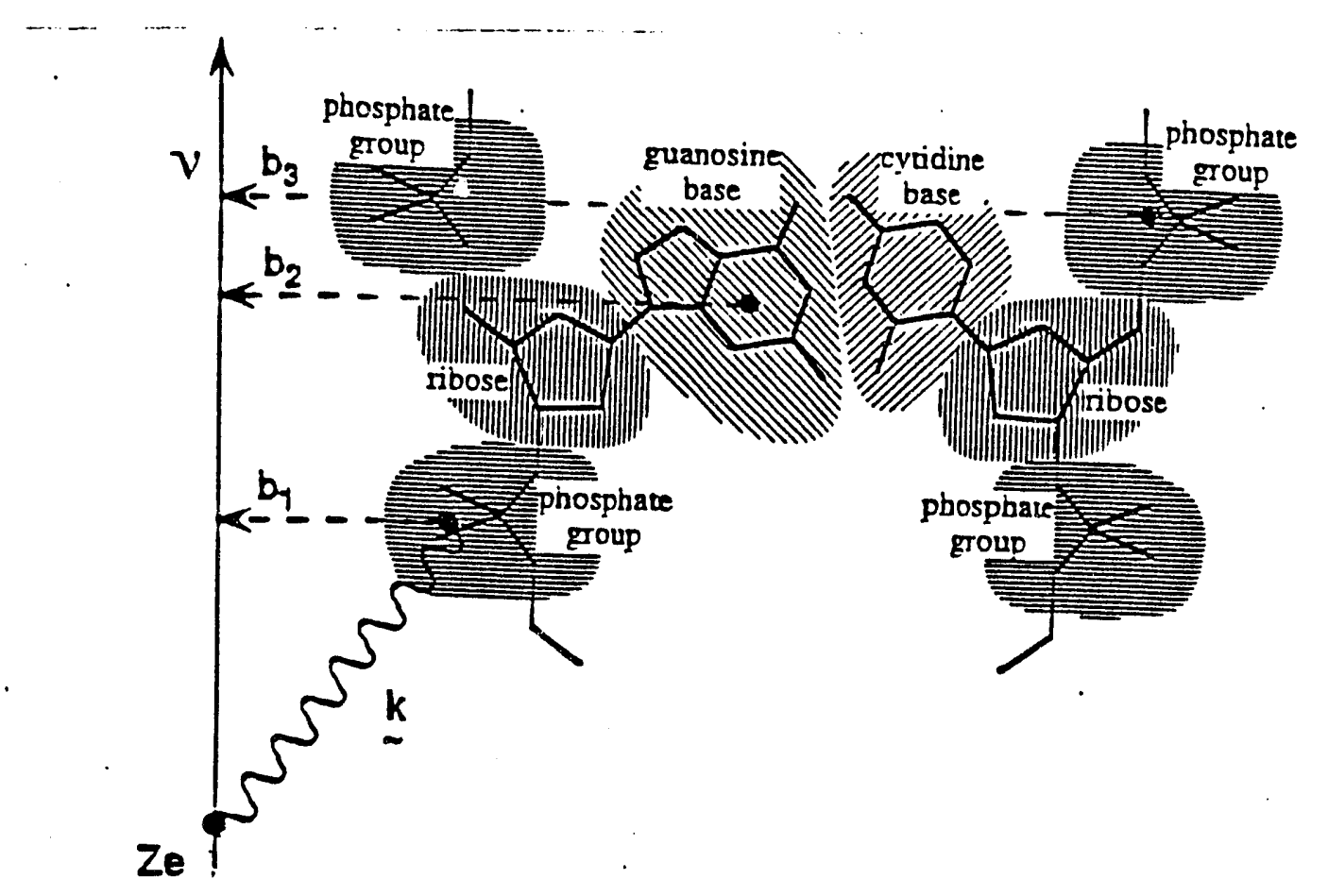

Figure 6. Spatial relationship between functional groups of DNA and a particle trajectory that passes close to the double helix.

parameter distribution for each group by methods similar to those discussed above making use of optical data on the groups in isolation but with the constraint that the model remain consistent with DNA photoabsorption data ${ }^{(10)}$. This approach will allow us to include the internal structure of DNA in determining the impact parameter to a given functional group, which we argue on the basis of Fig.(5) will have a major effect on the probability to transfer energy to the group in many of the cases of current interest. Furthermore, this extension of the current theory will permit the different ionization thresholds of the components of $\mathrm{DNA}^{(16)}$ to be included in the model.

\section{SUMMARY AND CONCLUSIONS}

A semiempirical model has been presented from which differential inverse mean free paths can be calculated for electron- and ion-impact ionization of DNA in solution. The model includes the screening effect that results from the dielectric response of the aqueous medium, which strongly influences the cross section for ejection of low-energy electrons. An impact-parameter representation of the differential inverse mean free path has been developed that will allow simulation of energy transfer to DNA when the trajectory of the charged particle slowing down does 
not penetrate the electron cloud of the target DNA molecule. Since the scale of the impact-parameter probability distribution is roughly equal to Bohr's maximum effective impact parameter, $v / \omega$, this long-range mode of energy transfer is most important for ejection of low-energy secondary electrons by fast projectiles. In future applications of track-structure simulation to model direct induction of DNA lesions under cellular conditions, an extension of the current theory that allows for the internal structure of DNA will be required. An outline for future development of our model to meet this need has been presented.

\section{ACKNOWLEDGEMENTS}

This work was supported by the Office of Health and Environmental Research (OHER) of the United States Department of Energy.

\section{REFERENCES}

1. L. Udovicic, F. Mark, E. Bothe, and D. Shulte-Frolinde, Non-homogeneous kinetics in the competition of single-stranded calf-thymus DNA and lowmolecular weight scavengers of $\mathrm{OH}$ radicals: a comparison of experimental data and theoretical models, Int. J. Radiat. Biol. 59:677 (1991).

2. R.H. Shuler, A.L. Hartzell, and B. Behar, Track effects in radiation chemistry. Concentration dependence of the scavenging of $\mathrm{OH}$ by ferrocynide in $\mathrm{N}_{2} \mathrm{O}$-saturated aqueous solutions, J. Phys. Chem. 85:192 (1981).

3. S.G. Swarts, M.D. Sevilla, D. Becker, C.J. Tokar, and K.T. Wheeler, Radiationinduced DNA damage as a function of hydration: 1 . Release of unaltered bases, Radiat. Res. 129:333 (1992).

4. N. Bohr, The decrease in velocity of alpha rays, Phil. Mag. 25:10 (1913).

5. R.H. Ritchie, The interaction of charged particles with a Fermi-Dirac electron gas, Plys. Rev. 114:644 (1959). R.H. Ritchie, R.N. Hamm, J.E. Turner, and H.A. Wright, The interaction of swift electrons with liquid water, in: "Sixth Symposium on Microdosimetry," J. Booz and H.G. Ebert, eds., CEC EUR 6064, (1978).

6. R.H. Ritchie, R.N. Hamm, J.E. Turner, H.A. Wright, J.C. Ashley, and G.J. Basbas, Physical aspects of charged particle track structure, Nucl. Tracks Radiat. Meas. 16:141 (1989). R.H. Ritchie, A. Howie, P.M. Echenique, G.J. Basbas, T.L. Ferrell, and J.C. Ashley, Plasmons in scanning transmission electron microscopy electron spectra, Scanning Microscopy Supplement 4:45 (1990).

7. U. Fano, The formulation of track structure theory, int: "Charged Particle Tracks in Solids and Liquids," G.E. Adams, D.K. Bewley and J.W. Boag, eds., The Institute of Physics, London (1970).

8. J.S. Samson, Formulation of the binary-encounter approximation in configuration space and its application to ionization by light ions, Phys. Rev., 8:822.(1973). 
9. J.M. Heller, Jr., R.N. Hamm, R.D. Birkoff, and L.R. Painter, Collective oscillation in liquid water, J. Chem. Phys. 60:3483 (1974).

10. T. Inagaki, R.N. Hamm, and E.T. Arakawa, Optical and dielectric properties of DNA in the extreme ultraviolet, J. Chem. Phys. 61:4246 (1974).

11. R.H. Ritchie, C.J. Tung, V.E. Anderson and J.C. Ashley, Electron slowing-down spectra in solids, Radiat. Res. 64:181 (1975). C.J. Tung, R.H. Ritchie, J.E. Ashley, and V.E. Anderson, Inelastic interactions of swift electrons in solids, Oak Ridge National Laboratory Report ORNL-TM-5188, (1976).

12. H.A. Kramers, Collected Works, North Holland Publishing Co. Amsterdam (1955), p. 333. R. Kronig, J. Am. Opt. Soc. 12, 547 (1926).

13. M.W. Williams, R.N. Hamm, E.T. Arakawa, L.R. Painter, and R.D. Birkoff, Collective electron effects in molecular liquids, Int. J. Rad. Phys. Chem. 7, 95 (1975).

14. J.C. Ashley, Stopping power of liquid water for low-energy electrons, Radiat. Res. 89, 25 (1982). D.J. Brenner, Stochastic calculations of the fast decay of the hydrated electron in presence of scavengers: Tests of model consistency, Radiat. Phys. Chem. 32:157 (1988).

15. See ref.(5) above and R.H. Ritchie, R.N. Hamm, J.E. Turner, H.A. Wright and W.E. Bolch, Radiation Interactions and Energy Transport in the Condensed Phase, in: "Physical and Chemical Mechanisms in Molecular Radiation Biology," W.A. Glass and M.N. Varma, eds., Plenum Press, New York (1991). The scheme described in ref.(5) and used in OREC for representing the response function of water also includes relativistic and exchange corrections. In the present application of the OPWD model, such corrections are not important.

16. W. Sontag and K.F. Weibezahm, Absorption of DNA in the region of vacuum-uv (3-25 eV), Rad. and Environm. Bioplys. 12:169 (1975).

17. We have carried out calculations of the ejection of an electron from a charged center immersed in a water medium using the OREC scheme for representing the water dielectric function together with a somewhat different method for obtaining the impact parameter representation of the cross section. The results found are entirely consistent with those shown in Fig.(3).

18. W. Brandt and R.H. Ritchie, Primary processes in the physical stage, in: "Physical Mechanisms in Radiation Biology," R.D. Cooper and R.W. Wood, eds., National Technical Information Center, Springfield, VA (1974).

19. C.J. Alden and S.-H. Kim, Solvent-accessible surfaces of nucleic acids, J. MoL Biol. 132, 411 (1979).

20. J.F. Ward, Mechanisms of radiation action on DNA in model systems - Their relevance to cellular DNA, in: "The Early Effects of Radiation on DNA," E.M. Fieiden and P.O'Neill, eds., Springer-Verlag, Berlin (1991). 

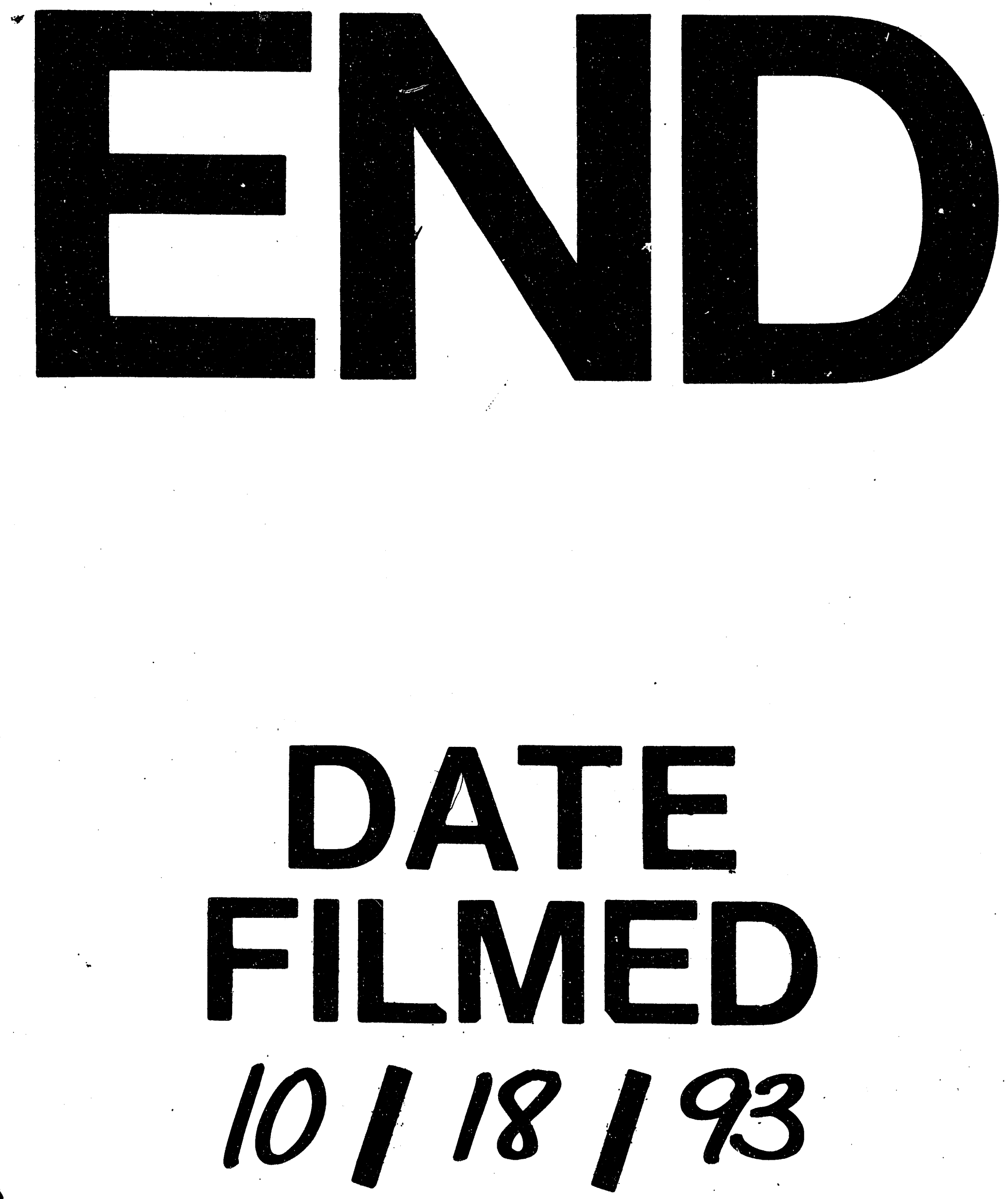